\title{
Segmentation and Reconstruction Techniques for Modeling of Blood Vessel
}

\author{
Vrushali Sudhir Taware \\ Dept. of Electronics and Telecommunication \\ Fr. C. Rodrigues Institute of Technology, Vashi \\ Navi Mumbai, India
}

\author{
Pranali C. Choudhari \\ Dept. of Electronics and Telecommunication \\ Fr. C. Rodrigues Institute of Technology, Vashi \\ Navi Mumbai, India
}

\begin{abstract}
Vascular diseases are nowadays one of the serious issues which have a huge impact on someone's life. Number of researchers at different universities as well as medical device manufacturers are working in this field for better understanding of the vascular characteristics. It is expected that three dimensional structure of blood vessels can provide comprehensive visualization of vessel geometry. This information will be useful in diagnosis and therapy related to vascular diseases. Several studies describe different numerical approaches to reconstruct a modeling of blood vessels closest to reality by using medical imaging. This paper gives extensive literature survey on segmentation and reconstruction techniques for artery modeling that uses various image modalities such as X Ray Angiography, Magnetic Resonance Angiography, Computed Tomography Angiography, Ultrasound etc. for the assessment of blood vessels.
\end{abstract}

\section{General Terms}

Artery, Biomedical Image Processing, Vascular diseases

\section{Keywords}

3D model; segmentation and detection; centerline extraction

\section{INTRODUCTION}

'Vascular' means a hollow container. It is a Latin word which is commonly used for the blood vessels in medical area. Blood vessels are the tubes through which blood is pumped throughout the body. It includes the arteries, veins and capillaries that carry blood to and from the heart. Arteries carry oxygen-rich blood from the heart to nourish every part of the body whereas Veins carry de-oxygenated blood back to the heart from muscles. This complete system is collectively called as vascular system and condition that affects this system is nothing but a vascular disease.

Vascular disease is an abnormal condition of the blood vessels. They may cause severe disability and death [1]. Vascular disease commonly occurs at sites of turbulent blood flow, such as when the direction of blood flow in the arteries changes abruptly. Few causes of these vascular diseases are listed below in Figure 1. Problems of the vascular system are common and can be serious. Thus, these diseases typically affect blood flow through the vessel. This may cause severe life threatening, problems with different symptoms such as ischemia in which these blood vessels are not able to provide enough blood to tissues [2]. In this case, there is a chance of generating dead tissues due to improper blood flow. This may leads to lose a limb or an organ. There is a need to study the characteristics of blood vessels like arteries in order to overcome these vascular diseases.

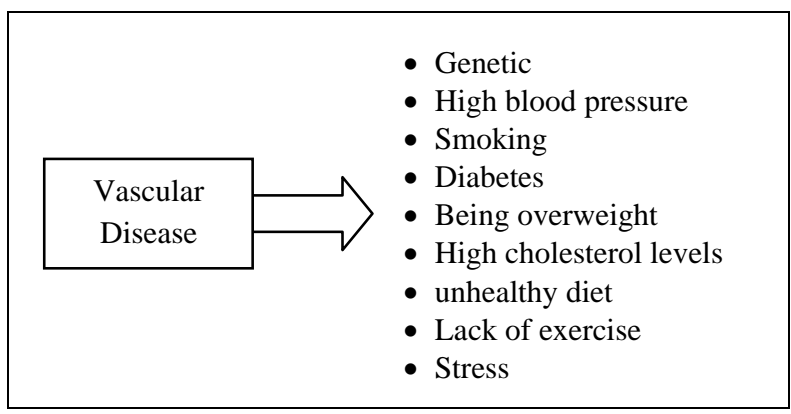

Fig 1: Causes of Vascular Diseases

For the assessment of vascular diseases, Angiograms have been used in medical area that uses an injection of iodine-rich contrast material as well as scanning. There is always a slight chance of cancer from excessive exposure to radiation in angiography. It may damage the skin, blood vessels and nerves [3]. Also, those medical procedures are uncomfortable in patient's view. There is a need of three dimensional model of artery (blood vessel) that retains all physiological as well as anatomical properties. This model will be useful not only in medical sectors but also for engineers and researchers. Thus this paper gives overview of the related work about the 3D reconstruction of artery implemented by different research groups.

The rest of this paper is organized as follows. Section 2 describes the advantages of $3 \mathrm{D}$ artery model. Section 3 outlines the related work on Segmentation and Detection algorithms for blood vessel and the centerline extraction techniques as well as different approaches for actual 3D reconstruction of artery. Summary and conclusion are given in Section 4

\section{THREE DIMENSIONAL MODEL}

Due to the ambiguity arising from a single projected view of a $3 \mathrm{D}$ elongated structure a single-plane angiogram alone is unreliable. It is beneficial to obtain three dimensional information on vessel morphology and hemodynamic to avoid mentioned issues. Such information can be used to support diagnosis, treatment planning, and outcome control of vascular diseases [4]. For modeling of surgical treatments for particular patients, vascular surgeons can use this 3D model. The additional information such as how well proposed surgical treatments restore blood flow to certain tissues or whether those treatments eliminate or introduce any unfavorable flow patterns will be provided by this $3 \mathrm{D}$ model [5].

Reconstructed 3-D data offers several advantages:

1. Helpful in the pre-stages of diagnosis.

2. Easier to combined with a reference model. 
3. Convenient for radiologist to deal with the large data sets.

4. More easy to scrutinize difference between current and previous exams of vascular system.

5. Simpler to understand and study (Compared to ECG, EMG such waves) for non-medical people.

6. Possible to study blood vessel's anatomy as well as physiology.

7. Improves service to referring physicians, since constructed 3D image can be attached to the radiology report. (These images illustrate the diagnosis and may even be shown to patients while discussing the condition and recommended treatment).

8. Helps in 3D Printing, device testing or the preparation of a surgery.

Hence, 3D reconstruction provides many important anatomical measurement that are either not available, or can't be accurately measured in $2 \mathrm{D}$. It provides better and clear visualization allowing people without extensive training to understand vessel geometry [6],[7]. It saves reviewing time for doctors since $3 \mathrm{D}$ reconstruction may be performed by a trained technician, and may also help visualize dynamics of the blood vessel that was not be demonstrated by previous methods. Furthermore, the accuracy of these models and the ease with which they are generated play an important role in determining the usefulness of these systems. This article describes a strategy for improving the process of geometric model construction for blood flow modeling applications.

\section{RELATED WORK}

\subsection{Segmentation and Detection Algorithms}

The algorithm for 3D reconstruction of blood vessel begins with this stage viz., the proper segmentation and detection of artery. This includes preprocessing of raw images (dataset) as well as identification and detection of vessel lumen (Figure 2).

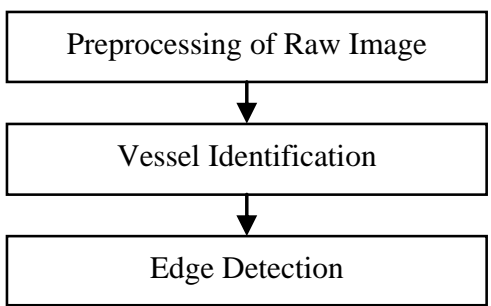

Fig 2: Pre-levels of Segmentation and Detection

There are several types of general segmentation algorithms, such as thresholding, watershed, region growing, classification and clustering [8]. These can work well with simple biomedical images with homogeneous regions. On the other hand, complex medical images such as CT images usually have many inhomogeneous regions. Pixels inside the vasculature do not necessarily have same intensity. There is always some noise that appears as pixels with low intensities. In these cases, general segmentation algorithms may fail. Therefore, more sophisticated algorithms have been developed.

Comparison of such 6 different methods for segmentation of artery in the pattern recognition class are given in [9]. The comparison parameters are used at input side (monoplane, biplane, rotational angiography dimensionality, enhancement methods etc.) and at output side (boundary, centerline, stenosis) and also they have mentioned whether the algorithm is effectively useful for $3 \mathrm{D}$ reconstruction or not. Those six methods are summarized in Table 1.

Table 1. Different Methods for Segmentation of artery

\begin{tabular}{|c|c|c|}
\hline \multicolumn{2}{|r|}{ Method } & Description \\
\hline 1 & $\begin{array}{l}\text { Multi scale } \\
\text { approaches }\end{array}$ & $\begin{array}{l}\text { Variations in image resolution are } \\
\text { used for segmentation. (low } \\
\text { resolution images are used to extract } \\
\text { large vessels while fine structures } \\
\text { are extracted at high resolution) }\end{array}$ \\
\hline 2 & $\begin{array}{l}\text { Region } \\
\text { growing } \\
\text { approaches }\end{array}$ & $\begin{array}{l}\text { Closely spaced pixels are considered } \\
\text { as same object and starting from } \\
\text { some seed point and adding new } \\
\text { pixels step by step to construct the } \\
\text { artery }\end{array}$ \\
\hline 3 & $\begin{array}{l}\text { Matching } \\
\text { filters } \\
\text { approaches }\end{array}$ & $\begin{array}{l}\text { Several filters are used in extraction } \\
\text { of the vessel contours } \\
\text { (Computational complexity and time } \\
\text { required is more) }\end{array}$ \\
\hline 4 & $\begin{array}{l}\text { Mathematical } \\
\text { morphology }\end{array}$ & $\begin{array}{l}\text { Basically two major steps - dilation } \\
\text { and erosion, morphological opening- } \\
\text { closing techniques are used }\end{array}$ \\
\hline 5 & $\begin{array}{l}\text { Skeleton } \\
\text { based } \\
\text { approaches }\end{array}$ & $\begin{array}{l}\text { Three methods : apply thresholding } \\
\text { and then object connectivity, } \\
\text { thresholding followed by a thinning } \\
\text { procedure, extraction based on graph } \\
\text { description }\end{array}$ \\
\hline 6 & $\begin{array}{l}\text { Ridge based } \\
\text { approaches }\end{array}$ & $\begin{array}{l}\text { By viewing an n-dimensional } \\
\text { grayscale (or color) image to be a } \\
\text { surface in an ( }+1) \text { dimensional } \\
\text { space by mapping intensity to height }\end{array}$ \\
\hline
\end{tabular}

Among these methods, 'Region growing method' is used widely for artery lumen detection. The user defines a seed point in the main artery and using connected pixels neighbor connectivity for lumen border detection is obtained [10]. One more method for luminal border detection is Connected component theory. In this volume is taken as input to identify artery bifurcation. This provides 3D volume \& gives approximated luminal border [11]. In [12], methods for vessel segmentation using an extended Kalman filter are presented. Those methods were fast and accurate, but had to be manually initialized with a seed point inside the vessel.

As mentioned above, the method 'region growing threshold connection' is applied for the segmentation of vascular tissues in [13]. The pixel value in a CT image is reported in Hounsfield units (a unit measuring the brightness of images that appear on CT scanner), which are calibrated to correspond to the attenuation of angiographic images measured within the particular sample of tissue. In [4], a method has been presented to sketch the vessel structures from a variety of medical images like CTA, MRA in less than $10 \mathrm{sec}$. They have found that this method is robust to typical problems occurring in 3D CTA and MRA images of the vasculature, such as branching vessels, calcifications, ringing artifacts and variations in intensity, shape, and size of the vessel. 
Number of rays is cast from a point in the plane perpendicular to the centerline. The casting of a ray is terminated once the border of the vessel is encountered. Further, modeling of the femoral artery considering as an ellipse has been presented in [14]. It consists of a real-time automatic artery detection method (eliminates manual intervention), a real-time vessel tracking method of the femoral artery (use of two Kalman filters, one for estimating the position of the vessel and another to estimate the shape), a real-time vessel 3D reconstruction method (possible to reconstruct bifurcations), and finally, a real-time vessel registration method (automatic and provides anatomical reference to the operator).

An accurate extraction of the artery from sequential CT slices as well as proper segmentation of vessel planes and plaque areas were the objectives of [15]. To identify and track arteries, extended regional maxima transformation (morphological transformation that suppresses the regional maxima in the gray scale image, whose height is bigger than the assumed threshold level) followed by a filtering process have been used. Using a disk shaped structuring element with 1 pixel radius in opening and closing operations, binary image is created. To select a proper artery (excluding bones and smaller vessels part) in CT image, masking operation is performed using previous slice. Comparison between the performance of a multi-slice $2 \mathrm{D}$ versus $3 \mathrm{D}$ cross-correlationbased radial and circumferential strain estimation has been presented in [16]. For detection of vessel lumen and calcified plaques segmentation is performed. Next, the lumen shape was adjusted to create an approximation of the formerly healthy lumen. The thickness of arterial wall was modeled by expanding the shape of the formerly healthy lumen, increasing its local radius with $30 \%$. A cylinder representing surrounding tissue was added and the model was meshed with vessel wall and surrounding tissue.

\subsection{Centerline Extraction}

After vessel detection, next important step to extract the centerline. The centerline is mathematically defined such that its perpendicular at any point intersects both vessel edges at equal distance from itself. Centerline extraction algorithm is shown in Figure 3. There are different approaches to determine it : (i) manually by the user, (ii) automatically [17] in a clearly defined region of interest, automatically from a given start point and possibly several end points, (iii) semi automatically [18], (iv) fully automatically [19]. Numerous techniques have been presented for this centerline extraction purpose. The most commonly used 13 coronary artery centerline extraction algorithms are summarized in [20].

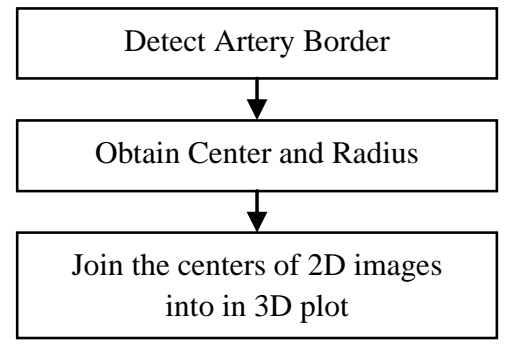

Fig 3: Centerline Extraction Algorithm

One of the centerline detection methods is the ridge-based algorithm. It regards a gray-scale image as a $3 \mathrm{D}$ elevation map where intensity ridges approximate the skeleton of the vasculature. Ridge points, which are local peaks in intensity, are first detected. Finally, the whole vascular structure is extracted by connecting neighboring ridge points. For quantification of stenosis using Computer tomography angiography is presented in [2].

Segmentation of coronary artery along with the centerline extraction is done by using Hessian filtering and the minimal path method. After obtaining two dimensional artery images and centerline, artery border is detected by ellipse fitting algorithm for edge detection, and consequently to compute the lesion's Area stenosis. Results after this experiment have shown that compared to Diameter stenosis, Area stenosis correlates better to the reference standard for stenosis quantification. A variety of approaches has been suggested to extract centerline of the artery from angiographic images of the vasculature. In [4], they have discussed two different classes of approaches which are summarized in Table 2.

Table 2. Centerline Extraction Approaches

\begin{tabular}{|l|l|}
\hline Approach & \multicolumn{1}{|c|}{ Description of Sub-approaches } \\
\hline $\begin{array}{l}\text { Indirect } \\
\text { approach }\end{array}$ & $\begin{array}{l}\text { Data is preprocessed before the centerline } \\
\text { detection (vessel axis). This requires } \\
\text { additional pruning and grafting methods } \\
\text { effectively this approach fails if the } \\
\text { segmentation is not accurate as well as if the } \\
\text { intensities of the vessels are not varying } \\
\text { smoothly. }\end{array}$ \\
\cline { 2 - 3 } & $\begin{array}{l}\text { Use of multi scale filters that generates } \\
\text { maxima in the middle of artery. Though this } \\
\text { method doesn't require pre-segmentation } \\
\text { and applicable for varying width of vessels, } \\
\text { it has high computational load. }\end{array}$ \\
\hline $\begin{array}{l}\text { Direct } \\
\text { approach }\end{array}$ & $\begin{array}{l}\text { Extraction of the centerline directly from the } \\
\text { dataset by using mean of the intensities. }\end{array}$ \\
\cline { 2 - 3 } & $\begin{array}{l}\text { Extraction of the centerline by convolution } \\
\text { with a Gaussian kernel in multiple } \\
\text { directions. }\end{array}$ \\
\cline { 2 - 3 } \\
$\begin{array}{l}\text { Extraction of the centerline by circle or } \\
\text { ellipse fitting algorithm. }\end{array}$ \\
\cline { 2 - 2 } $\begin{array}{l}\text { Such indirect methods are not able to cope } \\
\text { with large variations in the size of the } \\
\text { objects tracked. To overcome this, an } \\
\text { iterative tracking process [4] is used which } \\
\text { is guided by locally computed gravity point. }\end{array}$ \\
\hline
\end{tabular}

The coronary artery motion modeling from 3D cardiac CT sequence also needs the initial stages of artery extraction. This is done by using contrast limited adaptive histogram equalization in [3] to improve the visibility of image sequences. Smoothing is followed by this step while preserving edges and other salient features. For identification of vessel axis a multi scale Hessian-based vessel enhancement filter is used with 2nd order derivatives of the image intensity. Then the motion tracking is compared using two different methods. In another artery tracking algorithm the local shape information is captured by using sphere-based geometric model to represent each vessel segment. The vessel centerline is represented by the sphere centroids and the vessel surface is represented as the envelope of these spheres. This method uses a single initial seed point at the top of the tree and recursively tracks every vessel branch based on a Sequential Monte Carlo framework [21]. 


\subsection{D Re-construction Techniques}

Without any additional information or calibration, it is not possible to determine the absolute size of a vessel from single plane angiographic images because no information about the magnification is available. It is also not possible to reconstruct the $3 \mathrm{D}$ vessel geometry from single plane angiographic images because no information about the depth of the vessels is available. Thus, using segmentation and detection algorithms these information is extracted which is further used for $3 \mathrm{D}$ construction as shown in Figure 4.

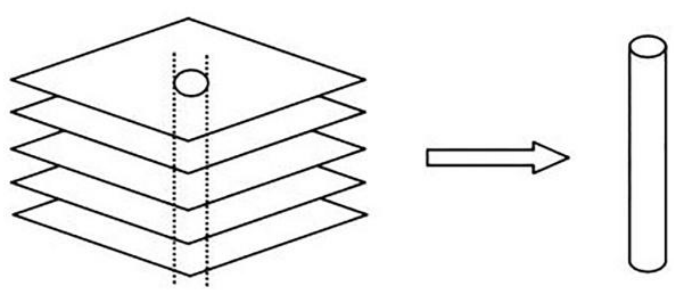

Fig 4: Three dimensional reconstruction of artery from 2D images

For construction of ascending, arch and descending aorta using CTA database, a semi-automated method have been presented in [12]. To determine aortic centerline coordinates, circle fitting algorithm is used. These coordinates follows toroidal path for the curvilinear portion and axial planes for descending aorta. This algorithm reconstructs oblique planes orthogonal to the centerline direction for area covered. Vessel borders are identified by circle fitting process. Using these initial stages, further aortic dimensions are estimated such as vessel diameter, inner volume and vertical length of aorta. Also the shape of aorta viz., arch width, height and curvature are calculated. Due to semi-automated method, manual intervention is required.

IVUS viz., Intravascular Ultrasound (non invasive) technique is better than CTA (invasive) is explained in [22]. Canny operator is used for edge detection and using Hough Transform arterial walls are extracted. After radial search, 3D presentation is made by either Oblique projection or the combination of Image space and Depth storing approach. Though this method gives approximation even when wall data is missing, ultrasound images are difficult at diagnostic stage. The new methodology that allows reliable segmentation of the magnetic resonance images (MRIs) for accurately automated 3D reconstruction of the carotid arteries has been discussed in [11]. This approach includes Image acquisition and preprocessing and automatic characterization of plaque composition. For 3D visualization Triangulation algorithm (connect most distal borders) is used. The another approach for computation of vascular flow resistance of coronary artery with the aim of assessment of stenosis impact on blood flow is discussed in [23]. The required preparatory reconstruction of coronary artery from CTA dataset was described as: (i) Preprocessing of raw CTA dataset (ii) Hessian based vessel enhancement with Frangi's filter (iii) Intensity based thresholding and localization of artery (iv) segmentation of vessel (active contour method) (v) Centerline extraction and branch detection and finally the branch resistance is calculated.

In some literatures, a three dimensional coronary artery model is constructed using CTA images and compared the reconstructed model with IUVS ground truth for validation [24]. One of the algorithms used in those have work of flow as follows: preprocessing of images, removal of artifacts, identification of vessel by image thresholding, then Rough artery border detection, defining centerline, detection of lumen \& outer wall border, plaque region estimation and finally, 3D construction. The results are matched perfectly with the IUVS data for validation of algorithm [10]. In computer assisted diagnosis of coronary artery diseases, vascular structure identification and matching in sequential angiographic images obtained at different angles have great significance. The 3D coronary artery model is reconstructed using theory of binocular stereo vision in [25]. They have worked on matching energy function between blood vessels that are constructed on the basis of local geometry and projection properties. A new method for $3 \mathrm{D}$ reconstruction of artery using the fusion of biplane angiograms and IVUS images is discussed in [26]. Automatic ROI detection in Ultrasound images is performed along with the extraction of catheter path in angiographic images. Then these IVUS frames are placed on catheter path (acting as a centerline) and relative axial twist is estimated. Finally, the absolute orientation of first IVUS frame is determined and accordingly $3 \mathrm{D}$ artery is constructed. Thus, there are several different ways that are used by researchers for 3D reconstruction of artery in their work which are summarized in Table 3.

\section{CONCLUSION AND SUMMARY}

The segmentation of blood vessels in angiograms has been heavily researched in recent years. According to the researchers and scientists it has been proved that the accurate extraction of arterial characteristics has great significance in recent computerized systems which are used in observation and diagnosis of vascular diseases. Even though many segmentation and reconstruction techniques have been proposed in this area, there is still room for improvement in arterial segmentation. In addition to this there are several different aspects which need to be considered such as innerouter volume, thickness of walls, plaque region etc. Therefore, this paper reviewed both early and recent papers which focus on different types of arterial segmentation from various image modalities for different purpose.

Table 3. Summary of the Study

\begin{tabular}{|l|c|c|c|c|c|}
\hline \multicolumn{1}{|c|}{ Algorithm } & Year & $\begin{array}{c}\text { Image } \\
\text { Modality }\end{array}$ & $\begin{array}{c}\text { Artery } \\
\text { Section }\end{array}$ & Objective & Remark \\
\hline Zhou et al. [2] & 2015 & CTA & $\begin{array}{c}\text { Coronary } \\
\text { artery }\end{array}$ & $\begin{array}{l}\text { To quantify the severity of } \\
\text { coronary artery stenosis }\end{array}$ & $\begin{array}{l}\text { Quantification of stenosis by considering } \\
\text { 'area' is giving better results as compared to } \\
\text { conventional diameter stenosis as it has high } \\
\text { correlation with reference standard. }\end{array}$ \\
\hline $\begin{array}{l}\text { Athanasiou et al. } \\
\text { [9] }\end{array}$ & 2016 & $\begin{array}{c}\text { CTA, } \\
\text { IVUS }\end{array}$ & $\begin{array}{c}\text { Coronary } \\
\text { artery }\end{array}$ & $\begin{array}{l}\text { To construct 3D model of } \\
\text { artery and plaque } \\
\text { characterization }\end{array}$ & $\begin{array}{l}\text { Semi-automated, fast and accurate } \\
\text { algorithm } \\
\text { Re-constructed 3D model is validated }\end{array}$ \\
\hline
\end{tabular}




\begin{tabular}{|c|c|c|c|c|c|}
\hline & & & & & by comparing with IVUS groundtruth \\
\hline $\begin{array}{l}\text { Sakellarios et al. } \\
{[10]}\end{array}$ & 2012 & MRA & $\begin{array}{l}\text { Carotid } \\
\text { artery }\end{array}$ & $\begin{array}{l}\text { To construct 3D model of } \\
\text { artery and plaque } \\
\text { characterization }\end{array}$ & $\begin{array}{ll}\text { - } & \text { Fast algorithm } \\
\text { - } & \text { Allows quantitative analysis that } \\
\text { provides information about vessel wall } \\
\text { dimensions and plaque burden. }\end{array}$ \\
\hline Craiem et al.[11] & 2012 & CTA & Aorta & $\begin{array}{l}\text { To identify the best size } \\
\text { and shape descriptors of } \\
\text { the aging effects on } \\
\text { thoracic aorta morphology } \\
\text { in 3D. }\end{array}$ & $\begin{array}{l}\text { - Volume is better than other parameters } \\
\text { (length, diameter) to estimate the } \\
\text { changes in thoracic aorta size with aging } \\
\text { - Aortic arch width is the best choice of } \\
\text { descriptor of aging effects }\end{array}$ \\
\hline $\begin{array}{l}\text { Smistad et al. } \\
{[13]}\end{array}$ & 2016 & $\begin{array}{l}\text { Ultra } \\
\text { Sound }\end{array}$ & $\begin{array}{c}\text { Femoral } \\
\text { artery }\end{array}$ & $\begin{array}{l}\text { To create an assistant for } \\
\text { ultrasound guided to block } \\
\text { the femoral nerve. }\end{array}$ & $\begin{array}{l}\text { - Correct and automatic tracking of } \\
\text { femoral artery by creating 3D model. } \\
\text { - Algorithm creates an assistant for } \\
\text { ultrasound-guided regional anesthesia of } \\
\text { the femoral nerve }\end{array}$ \\
\hline Fekkes et al. [15] & 2015 & $\begin{array}{l}\text { Ultra } \\
\text { Sound }\end{array}$ & $\begin{array}{l}\text { Carotid } \\
\text { artery }\end{array}$ & $\begin{array}{l}\text { To compare the } \\
\text { performance of a multi- } \\
\text { slice 2D versus 3D cross- } \\
\text { correlation-based radial } \\
\text { and circumferential strain } \\
\text { estimation }\end{array}$ & $\begin{array}{l}\text { - Strain error significantly reduced from } \\
0.82 \% \text { in } 2 \mathrm{D} \text { to } 0.62 \% \text { using the } 3 \mathrm{D} \\
\text { technique } \\
\text { - Circumferential strain is found with the } \\
\text { decrease of } 0.46 \% \text { to } 0.41 \%\end{array}$ \\
\hline $\begin{array}{l}\text { Athanasiou et al. } \\
\text { [22] }\end{array}$ & 2012 & IVUS & NA & $\begin{array}{l}\text { To characterize plaque of } \\
4 \text { types - Dense calcium } \\
\text { (DC), Necrotic core (NC), } \\
\text { Fibrotic tissue (FT), Fibro- } \\
\text { fatty tissue (FFT) }\end{array}$ & $\begin{array}{l}\text { - } \\
\text { - Ullows identification of all plaques } \\
\text { wses geometrical parameters (vessel } \\
\text { more reliable plaque characterization. } \\
\text { - } \\
\text { Accuracy of algorithm is } 85.65 \%\end{array}$ \\
\hline Uus et al. [23] & 2015 & CTA & $\begin{array}{c}\text { Coronary } \\
\text { artery }\end{array}$ & $\begin{array}{l}\text { To compute vascular flow } \\
\text { resistance of } 3 \mathrm{D} \text { model, } \\
\text { assessment of stenosis } \\
\text { impact on blood flow }\end{array}$ & $\begin{array}{l}\text { - } \begin{array}{l}\text { Computed resistance information is } \\
\text { further useful in blood flow simulation }\end{array} \\
\text { - This method gives better result for } \\
\text { single vessel than whole tree }\end{array}$ \\
\hline
\end{tabular}

\section{REFERENCES}

[1] F. Yang, Z. G. Hou, S. H. Mi, G. Bin Bian, and X. L. Xie, "3D modeling of coronary arteries based on tubularenhanced CURVES segmented regions for robotic surgical simulation," 2013 IEEE International Conference on Robotics and Biomimetics, ROBIO 2013, December, pp. 2013-2018, 2013.

[2] J. Zhou et al., "Quantification of coronary artery Stenosis by Area Stenosis from cardiac CT angiography," Proceeding of the Annual International Conference of the IEEE Engineering in Medicine and Biology Society EMBS, vol. 2015-Novem, pp. 695-698, 2015.

[3] D. Ping, L. Risser, C. Metz, and L. Neefjes, "Coronary artery motion modeling from $3 \mathrm{~d}$ cardiac CT sequences using template matching and graph search," IEEE International Symposium on Biomedical Imaging: From Nano to Macro, IEEE 2010, pp. 1053-1056, 2010.

[4] O. Wink, W. J. Niessen, and M. A. Viergever, "Fast delineation and visualization of vessels in 3-D angiographic images," IEEE Transactions on Medical Imaging, vol. 19, no. 4, pp. 337-346, 2000.

[5] K. C. Wang, R. W. Dutton, and C. A. Taylor, "Improving geometric model construction for blood flow modeling: Geometric image segmentation and image- based model construction for computational hemodynamics," IEEE Engineering in Medicine And Biology, vol. 18, no. 6, pp. 33-39, 1999.

[6] G. Biglino et al., "3D-manufactured patient-specific models of congenital heart defects for communication in clinical practice: feasibility and acceptability," BMJ Open, vol. 5, no. 4, p. e007165, 2015.

[7] D. Parker, C. A. Taylor, and K. Wang, "Imaged based 3D solid model construction of human arteries for blood flow simulations," Proceeding of the 20th Annual International Conference of the IEEE Engineering in Medicine and Biology Society,vol. 2, no. 2, pp. 9981001, 1998 .

[8] Q. Yingyi, "Segmentation and reconstruction of 3D artery models for surgical planning," Diss. 2008.

[9] R. M. Tayebi et al., "Coronary artery segmentation in angiograms with pattern recognition techniques - A survey," Proceeding of the 2013 International Conference on IEEE in Advanced Computer Science Applications and Technologies (ACSAT) 2013, pp. 321326, 2014.

[10] L. Athanasiou et al., "Three-dimensional reconstruction of coronary arteries and plaque morphology using CT 
angiography - comparison and registration with IVUS," BMC Medical Imaging, vol. 16, no. 1, pp. 9, 2016.

[11] A. I. Sakellarios et al., "Novel methodology for 3D reconstruction of carotid arteries and plaque characterization based upon magnetic resonance imaging carotid angiography data," Elsevier, Magnetic Resonance Imaging, vol. 30, no. 8, pp. 1068-1082, 2012.

[12] D. Craiem, M. E. Casciaro, S. Graf, G. Chironi, A. Simon, and R. L. Armentano, "Effects of aging on thoracic aorta size and shape: A non-contrast CT study," Proceeding of the Annual International Conference of the IEEE Engineering in Medicine and Biology Society EMBS, pp. 4986-4989, 2012.

[13] Y. Sen, Y. Zhang, Y. Qian, and M. Morgan, "A comparison of medical image segmentation methods for cerebral aneurysm computational hemodynamics," IEEE 2011 4th International Conference on Biomedical Engineering and Informatics, vol. 2, pp. 901-904, 2011.

[14] E. Smistad and F. Lindseth, "Real-Time Automatic Artery Segmentation, Reconstruction and Registration for Ultrasound-Guided Regional Anaesthesia of the Femoral Nerve," IEEE Transaction on Medical Imaging, vol. 35 , no. 3, pp. 752-761, 2016.

[15] T. Markiewicz, M. Dziekiewicz, S. Osowski, R. Boguslawska-Walecka, W. Kozlowski, and M. Maruszynski, "Computerized system for quantitative assessment of atherosclerotic plaques in the femoral and iliac arteries visualized by multislice computed tomography," IEEE Transaction on Biomedical Engineering, vol. 62, no. 6, pp. 1490-1502, 2015.

[16] S. Fekkes et al., "2D versus 3D cross-correlation-based radial and circumferential strain imaging in a 3D atherosclerotic carotid artery model using ultrafast plane wave ultrasound," IEEE International Ultrasonics Symposium Proceedings, no. 12122, pp. 1-4, 2015.

[17] M. Goyal and A. P. Agrawal, "Global optimization for 3$\mathrm{D}$ reconstruction of coronary artery trees from angiographic image sequence," IEEE 2013 Fourth International Conference on Computer Communication and Networking Technology, pp. 1-5, 2013.

[18] O. Hajati, K. Zarrabi, R. Karimi, and A. Hajati, "CFD simulation of hemodynamics in sequential and individual coronary bypass grafts based on multislice CT scan datasets," Proceeding of the Annual International Conference of the IEEE Engineering in Medicine and Biology Society EMBS, pp. 641-644, 2012.

[19] I. Wächter, "3D reconstruction of cerebral blood flow and vessel morphology from x-ray rotational angiography," Diss. UCL (University College London), pp. 1-216, 2009.

[20] M. Schaap et al., "Standardized evaluation methodology and reference database for evaluating coronary artery centerline extraction algorithms," Medical Image Analysis, vol. 13, no. 5, pp. 701-714, 2009, in press.

[21] F. Zhao, R. Bhotika, M. Carlo, "Coronary artery tree tracking with robust junction detection in 3D CT angiography,” IEEE ISBI-2011, pp. 2066-2071, 2011.

[22] L. S. Athanasiou et al., "A novel semiautomated atherosclerotic plaque characterization method using grayscale intravascular ultrasound images: Comparison with virtual histology," IEEE Transactions on Information Technology in Biomedicine, vol. 16, no. 3, pp. 391-400, 2012.

[23] A. Uus, P. Liatsis, M. M. Jawaid, R. Rajani, and E. Benderskaya, "Assessment of Stenosis Introduced Flow Resistance in CCTA-Reconstructed Coronary Arteries," IEEE Xplore, pp. 313-320, 2015.

[24] S. Voros et al., "Prospective validation of standardized, 3-dimensional, quantitative coronary computed tomographic plaque measurements using radiofrequency backscatter intravascular ultrasound as reference standard in intermediate coronary arterial lesions," Elsevier, JACC Cardiovascular Intervention, vol. 4, no. 2, pp. 198-208, 2011.

[25] R. Xiao, J. Yang, J. Fan, D. Ai, G. Wang, and Y. Wang, "Shape context and projection geometry constrained vasculature matching for 3D reconstruction of coronary artery," Elsevier, Neurocomputing, vol. 195, pp. 65-73, 2016.

[26] C. V. Bourantas et al., "A method for 3D reconstruction of coronary arteries using biplane angiography and intravascular ultrasound images," Elsevier, Computerized Medical Imaging and Graphics, vol. 29, no. 8, pp. 597-606, 2005. 